\title{
Virtual Paths: Collaborations in Narratives of Cultural Heritage of São Carlos-SP
}

\section{SIGRADI2018 TECHNOPOLITICAS \\ xxii congresso da sociedade iberoamericana de gráfica digital 22th conference of the iberoamerican society of digital graphics \\ 07|08|09|novembro|2018 iau usp | são carlos | sp br}

\author{
Sandra Schmitt Soster \\ USP | Brazil | soster@sc.usp.br \\ Anja Pratschke \\ USP | Brazil | pratschke@sc.usp.br \\ Maria Vitória do Nascimento Inocêncio \\ USP | Brazil | maria.inocencio@usp.br \\ Maria Clara Cardoso \\ USP | Brazil | maria3.cardoso@usp.br
}

\begin{abstract}
The "Virtual Paths: Collaborations in Narratives of Cultural Heritage of São Carlos" Extension Project sought to analyze the use of the QR Code, which directs the user to an online portal containing information about historical buildings in São Carlos, to complement the dissemination of the city's cultural heritage. As a pilot case, during the year of research, QR Codes were developed for the São Carlos Railway Station. This process seeks to diversify the dissemination of information on heritage and promote greater engagement of the local community, as an invitation to an augmented tour through the monuments of the city.
\end{abstract}

Keywords: Cultural heritage; Digital media; QR Code.

\section{INTRODUÇÃO}

As ações de educação patrimonial, ou seja, as atividades voltadas à divulgação e à valorização do patrimônio cultural de uma população, foram instauradas no Brasil em 1980, pela museóloga Maria de Lourdes Parreiras Horta, a fim de suprir a necessidade de mediações entre os saberes científico e popular sobre a cultura. O projeto aqui apresentado partiu do entendimento de que, para preservar as diversas manifestações culturais que compõem a cidade, é preciso pensar em possibilidades renovadas de leituras e divulgação, já que as relações da população com o entorno urbano se transformam no decorrer do tempo concomitante às mudanças produzidas na tecnologia, no espaço e na sociedade.

Desde o início dos anos 2000, a comunicação e a divulgação da informação se diversificaram por meio das possibilidades de virtualização e digitalização espaciais. Os meios de comunicação vêm sendo utilizados como suportes de memória, em um "movimento geral de virtualização da informação e da comunicação" (LÉVY, 1999, p. 31). O espaço virtual se converteu em um grande acervo desterritorializado, essencial para a cidadania e integração da sociedade ao permitir o acesso da sociedade às fontes de informações imprescindíveis à manutenção de sua identidade. O estágio atual das mídias, especialmente promovido pela disseminação dos smartphones, possibilita um acesso ainda maior e a participação no entendimento e na promoção do patrimônio cultural.
$\mathrm{Na}$ área do patrimônio cultural, as diversas tecnologias digitais, conforme criadas, foram sendo incorporadas às estratégias das instituições de memória para o registro, a preservação e a divulgação (CAMERON; KENDERDINE, 2007). Como salienta Ulpiano Menezes (2007, p. 64), o espaço virtual deve ser entendido e experienciado como um território de exploração, de modo que propicie ao indivíduo o alargamento de seu conhecimento sobre o patrimônio. Ou seja, a representação virtual do patrimônio tem o potencial de ampliar o entendimento do bem físico pela complementação de informações, sua atualização contínua e ininterrupta, e a possibilidade de comunicação direta entre órgãos governamentais e comunidade local.

Nesse contexto, as mídias digitais são importantes instrumentos de divulgação e aprendizado sobre o patrimônio. A população deve ser chamada a participar ativamente na disseminação e preservação do patrimônio, de modo que o Estado e os cidadãos dividem direitos e deveres perante a construção e manutenção da memória e história.

\section{CONTEXTO MUNICIPAL}

São Carlos é uma cidade média brasileira, com cerca de 240 mil habitantes (Censo IBGE 2014) e polo educacional: com duas Universidades públicas, o campus sede da Universidade Federal de São Carlos (UFSCar) e o campus São Carlos da Universidade de São Paulo (USP).

De acordo com o arquiteto brasileiro Vladimir Benincasa (2008, p. 46), os Campos de Araraquara são inicialmente mencionados nos registros oficiais porque a região fazia 
parte do antigo "[...] caminho até Cuiabá, aberto em 1721 por Luís Pedroso de Barros e retomado em 1799 por ordem do então governador de São Paulo, D. Antônio Manoel de Mello". Já segundo o cientista social brasileiro Marcel Mano (2006, p. 86), tais terras somente foram ocupadas no começo do século XIX, no local onde atualmente se localiza a cidade de São Carlos, nas terras da Fazenda Pinhal, do Sr. Carlos José Botelho. Segundo o autor, em 1786, a Sesmaria do cirurgião-mor do Regimento de Voluntários Reais de São Paulo foi vendida ao capitão Carlos Bartholomeu de Arruda, que a cedeu por herança a Carlos José Botelho no ano de 1831, quando ela passou a ser povoada.

Segundo a doutora em sociologia brasileira Maria de Nazareth Baudel Wanderley (2004, p. 84), as fazendas de café, "[...] juntamente com as pequenas cidades, tiveram um importante papel na História do povoamento brasileiro, como pontos de apoio da civilizaçãó [...]". Tendo participado do período cafeeiro do estado, a cidade guarda diversas edificações históricas, conforme o mapa a seguir, que apresenta os bens tombados e de interesse histórico e cultural da Fundação Pró-Memória de São Carlos.

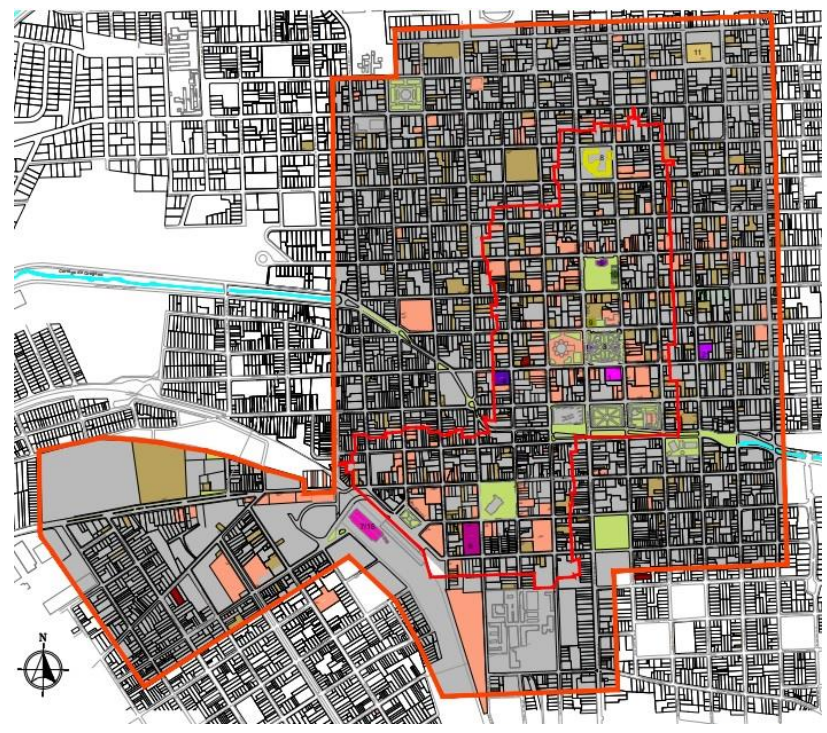

Figura 1: Poligonal histórica de São Carlos-SP (FUNDAÇÃO, 2016) Disponível em:

<http://www.promemoria.saocarlos.sp.gov.br/acervo-

files/historias-sc/mapa_poligonal_2016_imoveis_protegidos.pdf> Acesso em: Mai. 2018.

Atualmente, a divulgação do patrimônio cultural sãocarlense é realizada pela Fundação Pró-Memória de São Carlos por meio de material impresso, portal na Internet e rede social, e de informações em placas afixadas às edificações tombadas.

A Fundação publicou a série impressa "Percursos", iniciada em 2009. O projeto identificou, registrou e organizou informação sobre os imóveis de interesse histórico da cidade, que foram diagramados em quatro conjuntos de cartões-postais, cada qual com um mapa de localização. De acordo com a instituição, o projeto tinha o objetivo de "[...] estimular a proteção ao patrimônio, promovendo a visibilidade e acesso a informações históricas e técnicas dos imóveis da área central da cidade, inclusos na lista de bens declarados de interesse histórico-cultural do município" (FUNDAÇÃO, s.d., s.p.).

Como os meios de divulgação atualmente utilizados não possuem o alcance almejado pela instituição, a Fundação estabeleceu parceria com 0 grupo de pesquisa Nomads.usp para ampliar o número de cidadãos incluídos em suas ações patrimoniais, por meio da criação de interface que pudesse atingir o maior e mais heterogêneo público possível. Além disso, deveria possibilitar leituras múltiplas do patrimônio, a partir de entradas variadas, e incentivar a participação e colaboração dos usuários. Também foi requisitada a mínima aquisição possível de equipamentos complexos e custosos pela instituição, e indicada a intenção de acesso por meio de tecnologia móvel (smartphones e tablets), pois, como apontam os mexicanos Mabel Vazquez-Briseno et al. (2012, p. 219), o celular é o dispositivo mais amplamente utilizado hoje em dia.

Nesse sentido, o projeto está alinhado com o pensamento das arquitetas e urbanistas brasileiras Valquíria Sales Romero Marques e Renata Baesso Pereira (2014, p. 4): "Se a melhor maneira de valorizar o patrimônio arquitetônico é através da educação patrimonial e a construção coletiva do conhecimento, acreditamos que o meio digital possa trazer uma contribuição importante. [...]". Entendendo-se a educação patrimonial em seu conceito mais amplo, ou seja, como o apontado pela arquiteta e historiadora Flávia Brito do Nascimento e pela geógrafa Simone Scifoni (2015) e pela Rede Paulista de Educação Patrimonial (s.d.), uma ação em que a comunidade local seja convidada a participar da reflexão sobre o que é o seu patrimônio.

\section{O PROJETO}

O projeto de extensão "Percursos Virtuais: Colaborações em Narrativas do Patrimônio Cultural de São Carlos" está sendo realizado por parceria firmada em 2017 pelo grupo de pesquisa Nomads.usp com a Fundação Pró-Memória de São Carlos. O objetivo principal foi analisar e implementar o uso do código de barras bidimensional QR Code para verificar a interação da população com o patrimônio cultural, em atividade a ser incluída nas políticas públicas de promoção do patrimônio cultural municipal, realizadas pela Fundação Pró-Memória de São Carlos. Os trabalhos realizados alimentam projetos de pesquisa então em andamento no grupo de pesquisa: uma iniciação científica sobre Qr Code para Memória e Preservação; uma iniciação científica sobre recursos digitais com potencial de utilização na área do patrimônio cultural; um doutorado sobre educação patrimonial; e um doutorado sobre colaboração das comunidades locais nas atividades realizadas pelos órgãos governamentais na área.

O projeto foi direcionado à utilização de código QR (sigla em inglês para Quick Response Code, ou código de resposta rápida) por sua facilidade de criação e de atualização e baixo custo de implantação e uso. Essa tecnologia pode ser utilizada com objetivos culturais, educacionais e científicos por possibilitar a organização e a divulgação de informações contidas nos acervos da instituição de maneira online, de forma rápida e ampla. Por se tratar de uma ação de fácil aprendizagem e de 
baixo custo, pode ser facilmente implementada e replicada em outros locais. Dessa forma, vem sendo muito utilizada para a área de patrimônio ao redor do mundo.

Além disso, como as cidades geralmente possuem áreas de interesse histórico com diversas edificações e locais de importância local, é possível utilizar o acesso à informação em tempo real para a criação de um itinerário para um passeio ao longo dessas áreas. Dessa forma, instiga-se a comunidade local a circular pela cidade em busca da história contida nos lugares, monumentos e edificações. Dois exemplos são apresentados nas Figuras 2 e 3.

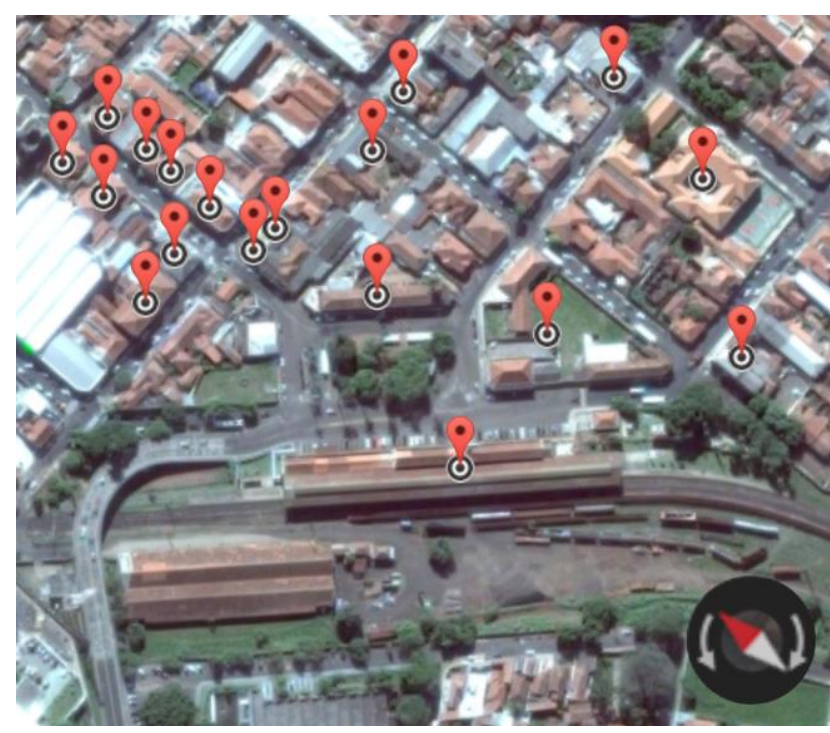

Figura 2: Exemplo de roteiros que pode ser criado na cidade de São Carlos-SP - próximo à Estação Ferroviária. Fonte: Fundação Pró-Memória de São Carlos sobre imagem do Google Maps.

Além disso, é importante incentivar a participação da comunidade local. O que será realizado por meio do envio de comentários, histórias e fotos, complementando a informação já coletada pela Fundação Pró-Memória de São Carlos. O conteúdo enviado pela comunidade é moderado pela instituição.

A tecnologia QR Code se aproveita de dispositivos já amplamente difundidos e que são de posse dos cidadãos, que possuem características técnicas apropriadas, tais como câmeras e acesso à Internet. Dessa forma, os celulares podem se tornar portais para integrar as realidades física e virtual. Como apontam VazquezBriseno et al (2012, p. 219, tradução nossa), "[...] O uso dessas tecnologias não só facilita a entrada de informação, mas também permite o uso dos celulares para interações entre pessoas, lugares e coisas, melhorando a usabilidade e utilidade desses dispositivos".

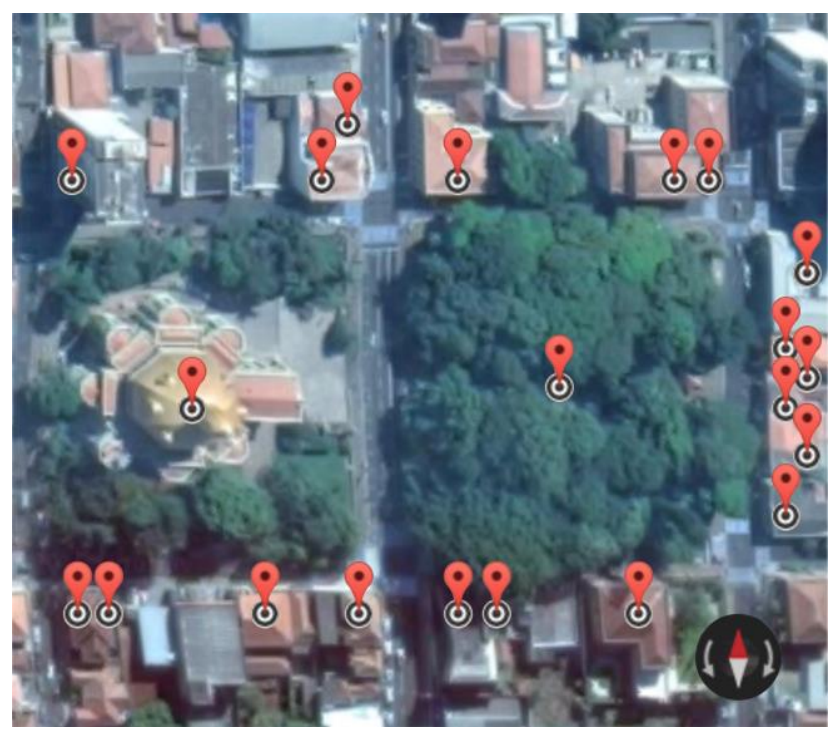

Figura 3: Exemplo de roteiros que pode ser criado na cidade de São Carlos-SP - nas redondezas da Praça da Catedral.

Fonte: Fundação Pró-Memória de São Carlos sobre imagem do Google Maps.

Nesse sentido, entende-se que tal tecnologia possibilita uma ampliação dos bens patrimoniais ao responder ao que Clifford (1999 apud MENEZES, 2007) chama de zona de contato: um lugar de encontro entre o indivíduo e o bem cultural. Onde comunidade local e especialistas podem acumular e trocar informações por meio da entrada e da saída de dados. De modo que espera-se que o espaço criado seja um local que propicie a formação de laços afetivos da comunidade com os elementos de sua história, pois o sentimento de pertencimento justifica a permanência do patrimônio.

\section{A TECNOLOGIA}

Segundo Vazquez-Briseno et al (2012, p. 222), o QR Code foi desenvolvido em 1994 pela Denso Wave Corporation. É uma tecnologia que contém informação em duas direções e, por isso, permite o armazenamento de 7.089 números, 4.296 alfanuméricos, 2.953 binários ou 1.817 kanjis (VAZQUEZ-BRISENO, et al, 2012).

O projeto de extensão "Percursos Virtuais: Colaborações em Narrativas do Patrimônio Cultural de São Carlos" analisou o uso do QR Code, acoplada a uma plataforma online de informações, como interface de divulgação do patrimônio cultural de São Carlos. Tendo como piloto a Estação Ferroviária de São Carlos, a parte técnica do projeto foi desenvolvida por uma aluna de graduação da área de tecnologia da informação em estrutura estática, por meio de conteúdo adicionado diretamente em código fonte. Contudo, a manutenção da plataforma precisaria ser realizada diretamente nos servidores por meio de linguagem técnica, o que mostrou-se um limitador para os funcionários da Fundação, futuros administradores da plataforma, que não possuem conhecimento na àrea. Nesse sentido, a exigência de formação específica para a manutenção do projeto foi identificada como uma dificuldade futura, que engessaria a atualização e a criação de novos conteúdos. 


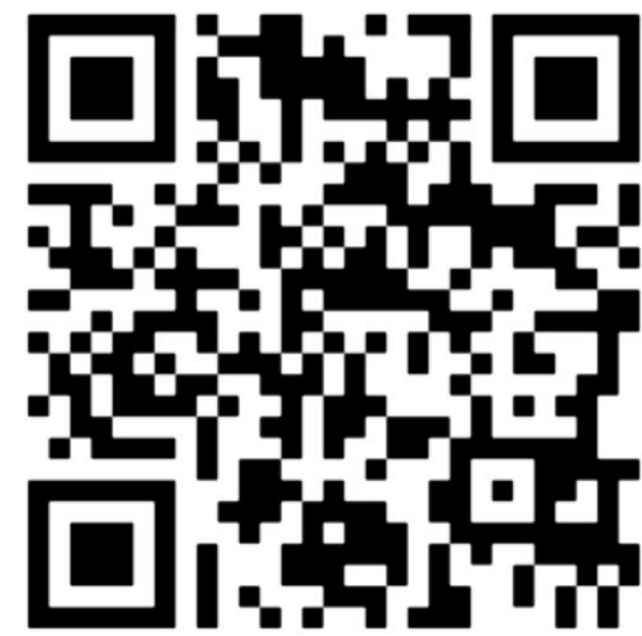

Figura 4: QR Code de um dos pontos fixados na Estação Ferroviária de São Carlos. Fonte: Arquivo do projeto.

Dessa forma, optou-se pela utilização da plataforma wordpress como intermediária entre o código e 0 administrador, tornando a manutenção, a edição e a criação de conteúdo mais intuitivas e acessíveis. A migração e adaptação do código já criado foram realizadas, tornando a plataforma mais dinâmica. Agora, os futuros administradores têm controle sobre o formato e a aparência do conteúdo, pois o wordpress possibilita personalização.

Com a estrutura pronta, foram criados os QR Codes, que serão acessados via dispositivos móveis, através de um leitor de QR Code instalado pelo visitante em seu próprio dispositivo móvel, de modo que o usuário acesse o site sem necessidade de digitação da url do mesmo. Os QR Codes são fixados nos pontos correspondentes à informação neles contida e direcionarão o usuário para a página, com informações sobre 0 ponto que está visitando: perguntas e respostas relacionadas a curiosidades do bem tombado; rolando a página para baixo, maiores informações, a foto que embasou o desenho e uma caixa para comentários (Figura 5).

\section{O CONTEÚDO}

Inicialmente, a equipe de educadores da Fundação PróMemória de São Carlos definiu os pontos de interesse da Estação Ferroviária de São Carlos, onde foram instalados os QR Codes: 1. fachada; 2. relógio externo; 3. bilheteria; 4. portões de ferro da entrada; 5 . botequim; 6. bitola estreita; 7. Sociedade Protetora; 8. cabine de rádio; 9. cabine de sinalização; 10. armazém; 11. banheiro; 12. maria fumaça; 13. jardim; 14. Museu de São Carlos (ver Figura 6).

Em seguida, em paralelo à formulação do conteúdo escrito de cada ponto de QR Code pela Fundação Pró-Memória de São Carlos, o grupo de pesquisa Nomads.usp produziu desenhos para o fundo da interface tendo como base fotografias. Foi realizado levantamento fotográfico para as ilustrações que comporiam a interface, de acordo com os pontos de interesse. As fotos selecionadas foram redesenhadas com o auxílio de tablets e do aplicativo Paper.

O recurso digital disponibiliza diversas opções de formas de desenho, passando por diferentes tipos de traços a ferramentas de recorte e sobreposição. Tendo a imagem original como plano de fundo e funcionando como gabarito, foram selecionados os elementos de maior importância para a representação. Pela gama de recursos e a facilidade de criar e desfazer ações, o desenho amparado pela tecnologia mostrou-se mais ágil e fluido.

Por meio do redesenho, foi possível acrescentar às cenas elementos que facilitam o entendimento do uso dos espaços apresentados e que representam a escala humana. No caso da bitola estreita, no qual a forma atual difere da construção original, o desenho é um meio de reconstruir o cenário da época, permitindo a visualização de como era a bitola que foi retirada (ver Figura 3).
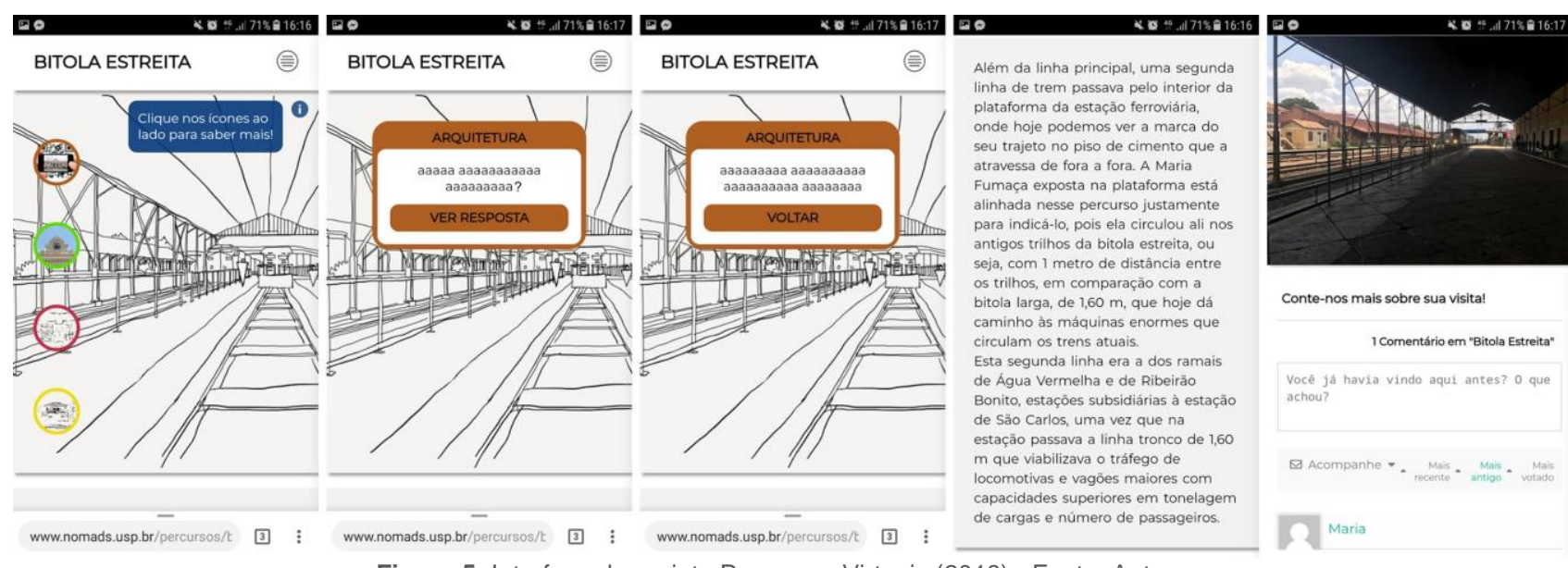

Figura 5: Interface do projeto Percursos Virtuais (2018). Fonte: Autoras.

As perguntas são acessadas na interface por meio de ícone coloridos, que direcionam para a resposta. Perguntas e respostas são curtas para despertar a curiosidade do usuário. Caso queira aprofundar o conhecimento sobre aquele assunto, há a opção Saiba + com a foto que embasou o desenho de fundo e maiores informações sobre aquele ponto. Os temas inicialmente 
pensados são: arquitetura, transporte, natureza, gastronomia e curiosidades.

A opção de comentário é essencial para a interface, já que por meio dela o usuário pode compartilhar suas impressões e informações históricas que possua, em textos ou imagens. Dessa forma, a plataforma também alimenta a produção de novos conhecimentos sobre o patrimônio representado, unindo o conhecimento técnico e o saber da comunidade local.

\section{CONSIDERAÇÕES}

$O$ acesso às informações através da leitura presencial dos QR Codes busca estimula a presença física e a relação com os bens patrimoniais no local, traçando um roteiro de aprendizagem que prevê a interação do usuário com o edifício da Estação Ferroviária e, posteriormente, com a área de seu entorno e outras regiões históricas da cidade. A tecnologia será utilizada em atividades desenvolvidas pelos educadores da Fundação Pró-Memória durante as visitas guiadas, além de facilitar o envio de comentários, histórias e fotos pela população, a fim de complementar o conhecimento sobre os pontos.

Além disso, a utilização dos QR Codes possibilita ao visitante traçar seu passeio individualmente, sem a necessidade de assistência da equipe de educadores do local. O que aumentará a facilidade e a autonomia de acesso a tal informação, consequentemente, ampliando também 0 número de pessoas alcançadas pela informação referente ao patrimônio da cidade.

Com a tecnologia em funcionamento, posteriormente, a equipe da Fundação Pró-Memória de São Carlos irá ampliar o número de QR Codes, tanto na própria Estação Ferroviária quanto distribuindo-os em outras edificações com a finalidade de criar um passeio interativo pela cidade. A médio prazo, o foco é a área em torno da
Estação Ferroviária (ver exemplo na Figura 2), e a longo prazo serão adicionadas edificações históricas da região central da cidades (ver exemplo na Figura 3), com a possibilidade de serem criados vários roteiros de passeio, organizados por temas ou estilos arquitetônicos, por exemplo.

Além disso, o conteúdo baseado em ambientes Web dos QR Codes pode ser atualizado ao longo do tempo sem grandes custos materiais. O que incentiva o público a visitar novamente o local em busca das novidades, que podem ser pelos funcionários da Fundação, e divulgadas nas mídias sociais. Os próximos passos do projeto perpassam questões de baixo custo financeiro e de recursos pessoais. Os mais importantes são: a produção, fixação e manutenção de placas com os QR Codes nos pontos de interesse; a atualização do conteúdo online; e a moderação dos comentários em textos e fotos enviados pelos usuários.

As limitações de alcance do projeto são essencialmente duas e estão vinculadas aos usuários: a necessidade de instalação de aplicativo leitor de QR Code e a conexão à rede Internet no momento da visita. São questões de fácil resolução, pois um aplicativo simples de leitura de QR Code possui pequeno tamanho (cerca de $2 \mathrm{Mb}$ ) e a maioria das pessoas utiliza pacotes de dados móveis em seu cotidiano.

Uma vez que se percebe uma limitação da tecnologia $Q R$ Code em relação à colaboração da comunidade local, o próximo passo da parceria estabelecida entre a Fundação Pró-Memória de São Carlos e o grupo de pesquisa Nomads.usp buscará a criação de plataforma online para colaboração entre órgão público, universidades e comunidade local.

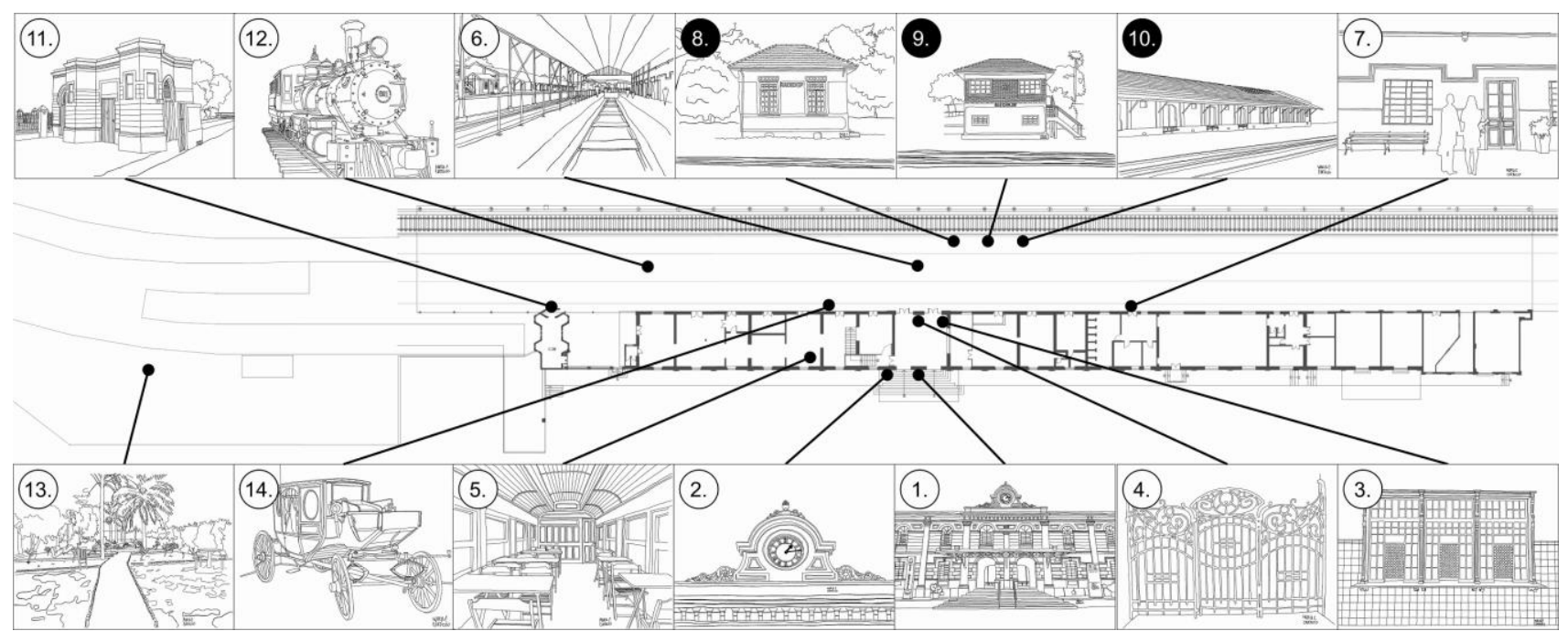

Figura 6: Pontos de QR Code do piloto da implantação na Estação Ferroviária. Os pontos 8, 9 e 10 referem-se a edificações do conjunto da estação, que não estão abertas à visitação. Por isso, seus QR Codes serão afixados no guarda-corpo da linha férrea, dentro da estação, de onde é possível avistar as referidas edificações. Fonte: Montagem de Sandra Schmitt Soster, com imagens do acervo da pesquisa e planta baixa da Fundação Pró-Memória de São Carlos.

O que inclui o georreferenciamento e a alimentação de conteúdo relacionado tanto ao patrimônio cultural oficializado e quanto às referências culturais não oficializadas. Também terá o intuito de colaboração entre os mesmos atores na tomada de decisões sobre o patrimônio cultural da cidade. 


\section{A EQUIPE}

Grupo de pesquisa Nomads.usp: Profa. Dra. Anja Pratschke, MSc. Jessica Aline Tardivo, MSC. Sandra Schmitt Soster, Maria Clara Cardoso e Maria Vitoria do Nascimento Inocencio.

Fundação Pró-Memória de São Carlos: Cláudia Regina Danella, Débora de Almeida Nogueira, Fábio Fontana de Souza, Mariana Lucchino e Rodrigo Santiago Peronti.

\section{AGRADECIMENTOS}

Agradecemos às agências financiadoras das pesquisas envolvidas neste projeto: Fundação de Amparo à Pesquisa do Estado de São Paulo (FAPESP) e à PróReitoria de Cultura e Extensão Universitária da USP.

\section{REFERÊNCIAS}

Benincasa, V. (2008). Fazendas paulistas: arquitetura rural no ciclo cafeeiro. 2v. Tese (Doutorado) - Escola de Engenharia de São Carlos, Universidade de São Paulo, São Carlos.

Cameron, F. and Kenderdine, S. (2007). Theorizing Digital Cultural Heritage: A Critical Discourse.Cambridge: MIT Press.

Fundação Pró-Memória de São Carlos (s.d.). Souvenirs. São Carlos: Fundação Pró-Memória de São Carlos. [online] Disponível <http://www.promemoria.saocarlos.sp.gov.br/index.php?optio $\mathrm{n}=$ com_content\&view=article\&id=125\&ltemid=166>.
Horta, M. L. P. (1999). Guia básico de educação patrimonial. Brasília: IPHAN: Museu Imperial.

Lévy, P. (1999). Cibercultura. São Paulo: Editora 34.

Mano, M. (2006). Os campos de Araraquara: um estudo de história indígena no interior paulista. Tese (Doutorado) Instituto de Filosofia e Ciências Humanas, Universidade Estadual de Campinas, Campinas.

Marques, V. S. R. and Pereira, R. B. (2014). Conhecer para preservar: o uso de QR Codes na educação patrimonial: o caso de Indaiatuba - SP. In: ENANPARQ, 3., São Paulo. Anais...

Meneses, U. T. B. (2007) Os museus na era do virtual. In: J. N. Bittencourt, M. Granato and S. F. Benchetrit (Org.). Museus, ciência e tecnologia. Rio de Janeiro: Museu Histórico Nacional. pp.48-70.

Nascimento, F. B. and Scifoni, S. (2015). O tombamento de Iguape como patrimônio nacional: novas práticas e políticas de preservação. PARC - Pesquisa em Arquitetura e Construção, Campinas, v. 6, n. 1, p. 26-38. Disponível em: <https://goo.gl/nVSQZt>. Acesso em: 30 Mar. 2018.

REPEP - Rede Paulista de Educação Patrimonial (s.d.). Princípios da educação patrimonial. Disponível em: <https://goo.gl/B9vHNB>. Acesso em: 6 Mai. 2018.

Vazquez-Briseno, M., et al. (2012). Using RFID/NFC and QRCode in Mobile Phones to Link the Physical and the Digital World. [s.I.]: Intechopen. Available at: $<$ https://cdn.intechopen.com/pdfs-wm/31056.pdf>

Wanderley, M. N. B. (2004). Olhares sobre o rural brasileiro. Raízes, 23 (1-2), p. 82-98. 